ITM Web of Conferences 4, 01002 (2015)

DOI: $10.1051 /$ itmconf/20150401002

(C) Owned by the authors, published by EDP Sciences, 2015

\title{
Initiation of erythropoiesis by BFU-E cells
}

\author{
Anass Bouchnita ${ }^{1}$, Nathalie Eymard ${ }^{2}$, Mark Koury $^{3}$, and Vitaly Volpert ${ }^{2, a}$ \\ ${ }^{1}$ Laboratoire de Biométrie et Biologie Evolutive, UMR 5558 CNRS, University Lyon 1, \\ 69622 Villeurbanne, France \\ ${ }^{2}$ Institut Camille Jordan, UMR 5208 CNRS, University Lyon 1, 69622 Villeurbanne, France \\ ${ }^{3}$ Vanderbilt University Medical Center, Nashville, TN, USA
}

\begin{abstract}
Erythropoiesis is the process of red blood cell production in the bone marrow. Terminal stages of human erythropoiesis occur in multicellular structures called erythroblastic islands (EBIs). EBIs contain up to several dozen erythroid cells of varying maturities organized around a central macrophage. Immature erythroid cells, burst forming units (BFU-E) circulate in blood and home to bone marrow, where they can have limited but random movement. When BFU-Es approach a macrophage, they divide producing colony forming units-erythroid (CFU-E), which are the next stage of erythroid differentiation. CFU-Es and their immediate progeny, proerythroblasts, can self-renew, differentiate into more mature cells or die by apoptosis. The BFU-E, CFU-E, and the subsequent erythroblast stages provide normal functioning of erythropoiesis. In this work we develop a hybrid discrete-continuous model in order to describe normal erythropoiesis in the bone marrow. Cells are represented as individual objects that move, divide, differentiate, die and interact with each other. We show how BFU-E cells initiate EBIs.
\end{abstract}

\section{Hybrid model of erythropoiesis}

Burst forming unit-erythroid (BFU-E) represents the earliest differentiation stage of hematopoietic cells committed solely to erythroid differentiation. BFU-Es circulate in blood, home to marrow, and have limited but random movement inside the marrow space where they divide and differentiate in response to growth factors produced by macrophages [1-3]. After BFU-Es divide and differentiate they become colony forming units-erythroid (CFU-Es). CFU-Es and their progeny, the erythroblasts, form erythroblastic islands around the macrophages. Thus, BFU-E cells initiate erythroblastic islands in the bone marrow. Their role is particularly important when the number of erythroblastic islands should be increased during stress erythropoiesis (anemia, hypoxia, treatment of blood diseases).

A hybrid discrete-continuous model of erythropoiesis was developed in [4-11]. We will briefly review the model below in this section. Intracellular protein concentrations that determine cell fate are described by ordinary differential equations. Concentration distributions in the extracellular matrix of substances that affect the intracellular protein concentrations are described by partial differential equations. Cells are considered as individual objects that can move, divide, differentiate, or die by

\footnotetext{
a e-mail: volpert@math.univ-lyon1.fr
}

This is an Open Access article distributed under the terms of the Creative Commons Attribution License 4.0, which permits unrestricted use, distribution, and reproduction in any medium, provided the original work is properly cited. 
apoptosis. In this work we consider the previous model to which we add one more cell type, the BFU-E, and we show how BFU-Es initiate erythroblastic islands.

Intracellular regulation. In the simplified model of intracellular regulation, the fate of erythroid progenitors is determined by four main factors: activated glucocorticosteroid receptor (z), BMPR4 receptor (u), GATA-1 (v) and activated caspases (w). Their concentrations are determined by the following system of ordinary differential equations [11]:

$$
\frac{d z}{d t}=k_{n e w}, \quad \frac{d u}{d t}=\lambda_{1}+b_{0}, \quad \frac{d v}{d t}=\lambda_{2}-b_{2} z v, \quad \frac{d w}{d t}=\lambda_{3}-b_{3} z w .
$$

The parameters $\lambda_{1}, \lambda_{2}$ and $\lambda_{3}$ in these equations are not fixed. They depend on the concentrations of extracellular substance. We use linear approximations for these functions:

$$
\lambda_{1}=\alpha_{1} G+\lambda_{10}, \quad \lambda_{2}=\alpha_{2} E+\lambda_{20}, \quad \lambda_{3}=\alpha_{3} F_{L}+\lambda_{30}
$$

Here $\alpha_{1}, \alpha_{2}, \alpha_{3}, \lambda_{10}, \lambda_{20}$ and $\lambda_{30}$ are some constants, $G$ is the concentration of growth factor BMP4 produced by macrophages, $F_{L}$ is the concentration of Fas-ligand produced by erythroid cells, $E$ is the concentration of hormone erythropoietin which is supposed to be constant throughout this work. We will return to the extracellular regulation in the next paragraph.

The fate of erythroid progenitors is determined by concentrations of intracellular substances. If the concentration $w$ of activated caspases exceeds some critical value $w_{c r}$, then the cell dies by apoptosis. If $w$ remains less than the critical value, then the cell will divide at the end of cell cycle, giving rise to two daughter cells similar to the mother cell (self-renewal) if $u>v$. Otherwise the daughter cells will be more mature than the mother cell (differentiation). We do not consider all intermediate stages of differentiation and suppose that erythroid progenitors differentiate directly into reticulocytes. The latter stay in the bone marrow for a period of time, then they leave it before entering the blood flow where they become mature erythrocytes.

Extracellular regulation. Intracellular regulation of erythroid progenitor cell fate is influenced by cell-cell interaction, growth factor cell fate produced by the surrounding cells and by hormones delivered to the bone marrow by the blood flow. The main two factors of extracellular regulation considered in the model are Fas-ligand $\left(F_{L}\right)$ and growth factor BMP4 whose concentration is denoted by $G$. Fas-ligand is produced by late-stage erythroblasts and the reticulocytes formed from them in humans and early-stage erythroblasts in mice. If the concentration of Fas-ligand is sufficiently high, then the cell will die by apoptosis; for intermediate values it will differentiate. The growth factor is produced by macrophages. It stimulates self-renewal of erythroid progenitors [16]. We use reaction-diffusion equations to describe the concentration of these two proteins:

$$
\begin{gathered}
\frac{\partial G}{\partial t}=D_{1} \Delta G+W_{1}-\sigma_{1} G ; \\
\frac{\partial F_{L}}{\partial t}=D_{2} \Delta F_{L}+W_{2}-\sigma_{2} F_{L} .
\end{gathered}
$$

Here $D_{1}, D_{2}$ are diffusion coefficients and $W_{1}, W_{2}$ are the rates of production of the corresponding factors. These functions are proportional to the concentrations of the corresponding cells. The last terms in the right-hand sides of these equations describe degradation of these substances.

Cell displacement. Since cells divide and increase their number, they can push each other resulting in cell displacement. In order to describe mechanical interaction between cells, we restrict ourselves to 
the simplest model where cells are presented as elastic spheres. Consider two elastic spheres with centers at the points $x_{1}$ and $x_{2}$ and with the radii, respectively, $r_{1}$ and $r_{2}$. If the distance $h_{12}$ between the center is less than the sum of the radii, $r_{1}+r_{2}$, then there is a repulsive force between them, $f_{12}$, which depends on the difference between $\left(r_{1}+r_{2}\right)$ and $h_{12}$. If a particle with center at $x_{i}$ is surrounded by several other particles with centers at the points $x_{j}, j=1, \ldots k$, then we consider the pairwise forces $f_{i j}$ assuming that they are independent of each other. This assumption corresponds to small deformation of the particles. Hence, we find the total force $F_{i}$ acting on the $i$-th particle from other particles, $F_{i}=\sum_{i} f_{i j}$. The motion of the particles can now be described as the motion of their centers by Newton's second law:

$$
m \ddot{x}_{i}+m \mu \dot{x}_{i}-\sum_{j \neq i} f_{i j}=0,
$$

where $m$ is the mass of the particle, the second term in the left-hand side describes the friction by the surrounding medium, and the third term is the potential force between cells. We consider the force between particles in the following form:

$$
f_{i j}=\left\{\begin{array}{cc}
K \frac{h_{0}-h_{i j}}{h_{i j}-\left(h_{0}-h_{1}\right)}, & h_{0}-h_{i}<h_{i j}<h_{0} \\
0, & h_{i j} \geq h_{0}
\end{array},\right.
$$

where $h_{i j}$ is the distance between the particles $i$ and $j, h_{0}$ is the sum of cell radii, $K$ is a positive parameter, and $h_{1}$ accounts for the incompressible part of each cell. This means that the internal part of the cell is incompressible. It allows us to control compressibility of the medium. The force between the particles tends to infinity when $h_{i j}$ decreases to $h_{0}-h_{1}$.

When a cell center crosses the boundary of computational domain, it is removed from the simulations. Under some conditions, cells can also be removed even if they are inside the computational domain. In particular, in the case of cell death or, in the case of blood cells in the bone marrow, if they leave bone marrow into the blood flow. When a new cell appears, we prescribe its initial position and initial velocity.

Cell properties and division. Biological cells considered in hybrid models are characterized by certain parameters such as their type, duration of cell cycle, initial radius and initial concentrations of intracellular proteins. These values are given to each cell at the moment of its birth. Some other cell properties are variable and they can change during the cell's life time. In particular, cell age, radius, position and current values of intracellular proteins. These values are recalculated at every time step.

Some cell properties can be given as random variables. For example, duration of cell cycle is considered as a random variable with a uniform distribution in the interval $[T-\delta T, T+\delta T]$. Initial protein concentrations can also be considered as random variables in some given range.

Cell fate is determined in two successive stages that begin at the end of cell cycle. At the first stage, they make a first choice between apoptosis and survival. If they survive and divide, they make a second choice between self-renewal and differentiation. In both cases, the choice is determined by the concentrations of intracellular proteins.

During cell cycle, cell radius grows linearly. It becomes twice the initial radius at the end of cell cycle. Then mother cell is replaced by two daughter cells. Geometrically, two circles with the initial radius are located inside the circle with twice larger radius. The direction of cell division, that is the direction of the line connecting the centers of daughter cells, is random. This is specific for cell division in hematopoiesis. In some other applications, direction of cell division can be prescribed.

Let us also note, that cell volume in the model is not preserved after division. The total volume of the daughter cells is half the volume of the mother cell before its division. Since cells in the bone marrow are sufficiently sparse, preservation of their volume during division is not essential. It can be important in some other applications where cell density is large and the forces acting between cells should be found more accurately. 


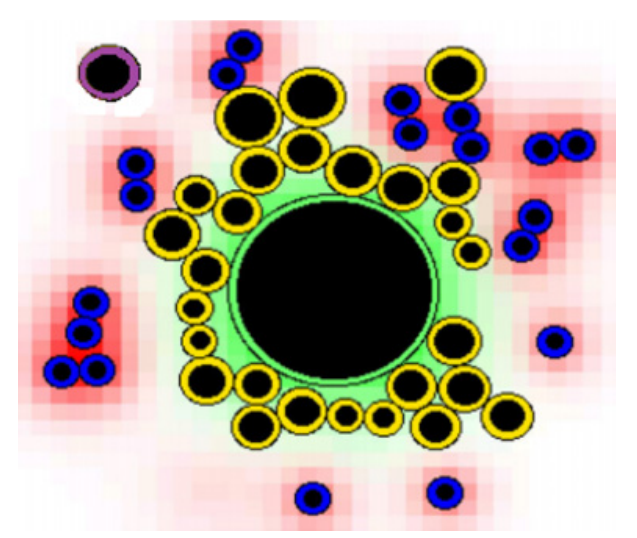

Figure 1. The structure of erythroblastic island. The green large cell in the middle is the central macrophage. Yellow cells are erythroid progenitors (CFU-E/erythroblasts), blue cells are reticulocytes, the violet cell is BFU-E. The red substance secreted by reticulocytes is Fas-Ligand. Growth factor produced by the macrophage is shown in green. Black circles inside cells show their incompressible part.

In the figures of the model and in actual erythropoiesis, the more mature erythroid cells are smaller than the earlier ones. This appears to be due to decreased cell cycle time during the cell divisions between CFU-E and orthochromatic erythroblasts.

\section{Initiation of erythroblastic islands}

Typical structure of erythroblastic islands is shown in Fig. 1. The central macrophage (big cell in the center) is surrounded by erythroid progenitors (yellow cells). Progenitors make a choice between selfrenewal, differentiation and apoptosis. This choice is determined by the intracellular regulation, and it depends on the extracellular substance, growth factor produced by the macrophage and Fas-ligand produced by the mature erythroblasts and the reticulocytes formed from them. Progenitors located close to the macrophage self-renew due to the growth factor. Progenitors close to reticulocytes die by apoptosis due to Fas-ligand. The cells located in between differentiate into reticulocytes.

After some time, reticulocytes leave the bone marrow into blood flow where they become mature erythrocytes. Since there is a small number of cells in erythroblastic islands, then random perturbations in cell cycle duration, apoptosis of erythroid progenitors and departure of erythrocytes can lead to extinction of the islands. This means that the macrophage remains without erythroid progenitors around it and new reticulocytes are not produced. Hence there should exist a mechanism of reinitiation of erythroblastic islands. Moreover, in the case of stress erythropoiesis (anemia, hypoxia) the number of erythroblastic islands can rapidly increase. This reinitiation mechanism is provided by BFU-E cells. They move randomly in the bone marrow. When they meet a macrophage, they divide producing CFU-Es and erythroid progenitors and restarting the erythroblastic island. It is shown that the macrophages in the marrow also move with both random and directional movement [17]. Thus, it might be possible for a randomly moving BFU-E to encounter a moving macrophage.

If the macrophage is surrounded by progenitors and reticulocytes, the BFU-E cell may not be able to approach it. However, when the macrophage is alone, the BFU-E can approach the macrophage and divide under the influence of growth factors produced by it.

In order to study this effect, we consider several macrophages without erythroid progenitors and introduces BFU-E cells (Fig. 2). The BFU-Es move in the bone marrow in a random way. Their trajectories are shown by magenta curves. When they approach the macrophages, they are influenced by the growth factor and begin to proliferate producing two CFU-Es. On the other hand, the erythroblasts 


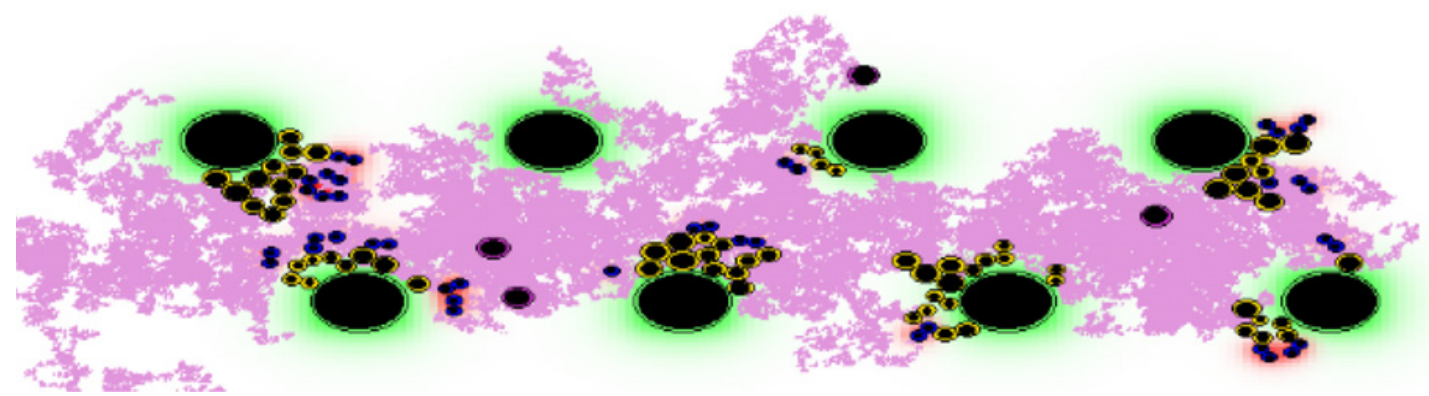

Figure 2. Burst forming unit (BFU-E) cells move randomly in the bone marrow. Magenta curve behind the cell shows its trajectory. When the cell approaches a macrophage (big cell), it divides and gives birth to two CFU-Es (erythroid progenitor). Progenitors divide creating erythroblastic island around the macrophage.

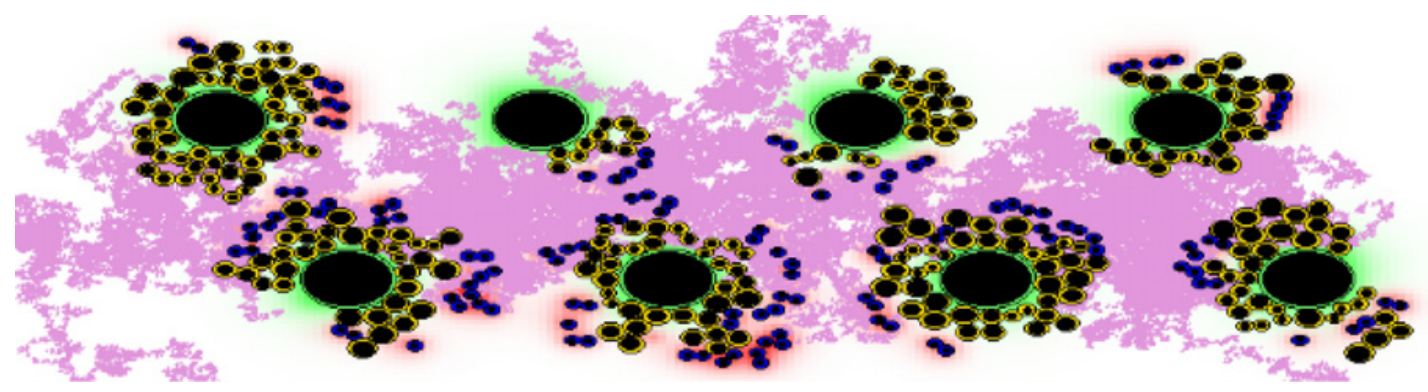

Figure 3. The same simulation as in Fig. 2 some time later. More erythroblastic islands emerge due to motion and division of BFU-E cells.

lose their adherence to the central macrophage, allowing them to be pushed out by other cells. As we discussed above, CFU-Es self-renew or differentiate, restarting the extinct island or forming a new one. Figure 3 shows the same simulation some time later when more islands are restarted.

The total numbers of erythroid progenitors and erythroblasts (Fig. 4A) as well as reticulocytes (Fig. 4B) in all islands are shown. They oscillate due to cell cycle, random perturbations, apoptosis of progenitors and departure of reticulocytes. Their average number increases due to the appearance of new islands. The values of parameters for these simulations are the same as in [11].

\section{Discussion}

We develop hybrid discrete-continuous multi-scale models in order to study red blood cell production in the bone marrow. We show in this work how immature cells, BFU-Es initiate erythroblastic islands in the case of their extinction or if new islands are required in the case of stress erythropoiesis.

Various factors can lead to the necessity to initiate new erythroblastic islands. They are related to the mechanisms of regulation of erythropoiesis and to randomness of some of its stages. First of all, the hormone erythropoietin (EPO) produced in the kidneys regulates the production of erythrocytes depending on the level of hemoglobin in blood $[13,15]$. If the EPO level is high, the production of red blood cells will be increased due to the down regulation of apoptosis of erythroid progenitors and due to the appearance of new islands. 
CFU-E and erythroblasts

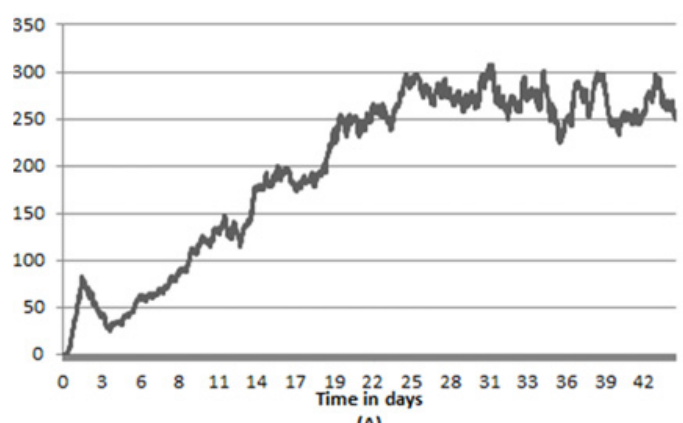

(A)

\section{Reticulocytes}

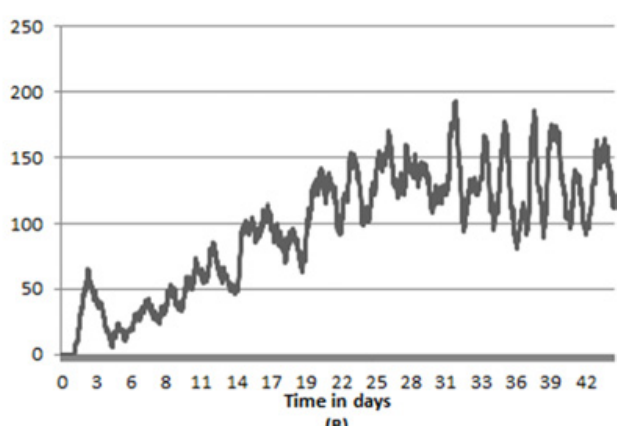

(B)

Figure 4. The total number of CFU-E and erythroblasts versus reticulocytes in EBIs over time for the same simulation as in Figs. 2 and 3.

Another possible situation is related to apoptosis of erythroid progenitors because of the high concentration of Fas-Ligand produced by increased numbers of mature erythroblasts and reticulocytes. All progenitors in the island can die and the island will become extinct. Destruction of erythroblastic islands massively occurs during some blood disorders such as multiple myeloma. It is a disease where immature plasma cells infiltrate the bone marrow and suppress erythroblastic islands by direct physical contact. Moreover, myeloma cells produce Fas-ligand and stimulate apoptosis of erythroid progenitors. Finally, proteins produced by myeloma cells can reduce EPO concentrations in blood [14] providing an additional factor suppressing erythroblastic islands. During treatment of multiple myeloma by chemotherapy, the majority of malignant cells are eliminated and erythroblastic islands start to recover. We will present the modelling of myeloma development and treatment in a forthcoming work.

BFU-E cells play an important role in erythropoiesis since they restart erythroblastic islands. They move in the bone marrow, and when they meet a macrophage, they divide due to some growth factors produced by it. As a result of this division, there are two CFU-Es that can self-renew and generate more differentiated erythroblasts. This is the mechanism of restarting erythroblastic islands or of initiating the new ones.

The hybrid model of erythropoiesis presented here has a number of limitations. The most essential one is related to the intracellular regulation of erythroid progenitors. The complete regulation is not known, it involves too many factors and the rate of intracellular reactions are basically unknown. At the actual stage of our knowledge, detailed modelling of intracellular regulation is not possible. This is the most important problem of the whole biological modelling. However, we take into account the most essential factors of the intracellular regulation that determine cell fate. This model shows realistic biological properties and it gives a good description of in vitro experiments and of the clinical data [11].

One of the important future applications of this model is related to modelling of development and treatment of various blood disorders, such as multiple myeloma or leukemia.

\section{References}

[1] V. S. Gallicchio, N. K. Hughes, B. C. Hulette, R. Della Puca and L. Noblitt, Basic fibroblast growth factor (B-FGF) induces early-(CFU-s) and late stage hematopoietic progenitor cell colony formation (CFU-gm, CFU-meg, and BFU-e) by synergizing with GM-CSF, Meg-CSF, and erythropoietin, and is a radioprotective agent in vitro. The International Journal of Cell Cloning, 9 (1991) 3, 220-232. 
[2] C. H. Dai, S.B. Krantz and K. M. Zsebo, Human burst-forming units-erythroid need direct interaction with stem cell factor for further development. Blood, 78 (1991) 10, 2493-2497.

[3] K. Muta and S. B. Krantz, Apoptosis of human erythroid colony-forming cells is decreased by stem cell factor and insulin-like growth factor I as well as erythropoietin. Journal of cellular physiology, 156 (1993) 2, 264-271.

[4] S. Fischer, P. Kurbatova, N. Bessonov, O. Gandrillon, V. Volpert and F. Crauste, Modeling erythroblastic islands: using a hybrid model to assess the function of central macrophage. Journal of theoretical biology 298 (2012) 92-106.

[5] P. Kurbatova, N. Eymard and V. Volpert, Hybrid Model of Erythropoiesis. Acta Biotheoretica, 61 (2013) 305-315.

[6] V. Volpert, N. Bessonov, N. Eymard and A. Tosenberger, Modèle multi-échelle de la dynamique cellulaire. In: Le vivant discret et continu, Nicolas Glade et d'Angelique Stephanou, Editeurs, Editions Materiologiques, 2013.

[7] N. Bessonov, N. Eymard, P. Kurbatova and V. Volpert, Mathematical modelling of erythropoiesis in vivo with multiple erythroblastic islands. Applied Mathematics Letters, 25 (2012) 9, 1217-1221.

[8] P. Kurbatova, S. Bernard, N. Bessonov, F. Crauste, I. Demin, C. Dumontet, S. Fischer and V. Volpert, Hybrid model of erythropoiesis and leukemia treatment with cytosine arabinoside. SIAM J. Appl. Math., 71 (2011) 6, 2246-2268.

[9] N. Bessonov, F. Crauste, S. Fischer, P. Kurbatova and V. Volpert, Application of Hybrid Models to Blood Cell Production in the Bone Marrow. Math. Model. Nat. Phenom., 6 (2011) 7, 2-12.

[10] N. Eymard and P. Kurbatova, Hybrid Models in Erythropoiesis and in Megakaryopoiesis. Math. Model. Nat. Phenom., 10 (2014) 1, in press.

[11] N. Eymard, N. Bessonov, O. Gandrillon, M. J. Koury and V. Volpert, The role of spatial organization of cells in erythropoiesis. Journal of mathematical biology (2014) 1-27.

[12] J. A. Chasis and N. Mohandas, Erythroblastic islands: niches for erythropoiesis. Blood, 112 (2008) 3, 470-478.

[13] L. O. Jacobson, E. Goldwasser, W. Fried and L. Plzak, Role of the kidney in erythropoiesis. Nature 179(4560), (1957), 633-4. doi: 10.1038/179633a0. PMID 13418752.

[14] Y. Beguin, M. Yerna, M. Loo, M. Weber and G. Fillet, Erythropoiesis in multiple myeloma: defective red cell production due to inappropriate erythropoietin production. British journal of haematology, 82 (1992) 4, 648-653.

[15] M. J. Koury, Erythropoietin: the story of hypoxia and a finely regulated hematopoietic hormone. Exp Hematol. (2005) 33, 1263-1270.

[16] M. M. Rhodes, P. Kopsombut, M. C. Bondurant, J. O. Price and M. J. Koury, Adherence to macrophages in erythroblastic islands enhances erythroblast proliferation and increases erythrocyte production by a different mechanism than erythropoietin. Blood (2008) 111, 1700-1708.

[17] T. Yokoyama, T. Etoh, H. Kitagawa, S. Tsukahara and Y. Kanna, Migration of erythroblastic islands towards the sinusoid as erythroid maturation proceeds in rat bone marrow. J. Vet. Med. Sci. 65 (2003) 4, 449-452. 